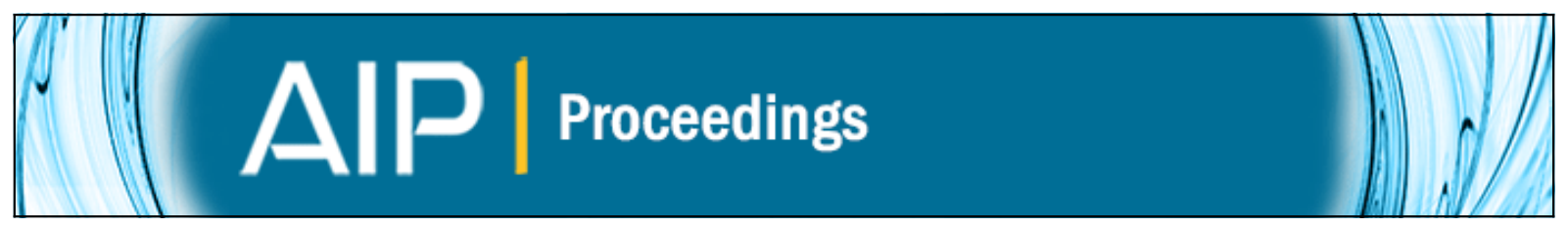

\title{
An Observational Perspective on Some Aspects of Early Stellar Nucleosynthesis
}

Christopher Sneden and James E. Lawler

Citation: AIP Conference Proceedings 990, 90 (2008); doi: 10.1063/1.2905680

View online: http://dx.doi.org/10.1063/1.2905680

View Table of Contents: http://scitation.aip.org/content/aip/proceeding/aipcp/990?ver=pdfcov

Published by the AIP Publishing

\section{Articles you may be interested in}

Cosmic Stellar Relics in the Galactic Halo

AIP Conf. Proc. 990, 459 (2008); 10.1063/1.2905664

Evolution and Nucleosynthesis of Low-Mass Metal-Poor AGB Models with C- and N-Enhanced Molecular Opacities.

AIP Conf. Proc. 990, 320 (2008); 10.1063/1.2905571

Nucleosynthesis in Metal-Free and Metal-Poor Stars

AIP Conf. Proc. 990, 283 (2008); 10.1063/1.2905564

Understanding the Characteristics of EMP Stars as Probes for the Early Universe: Stellar Evolution of Low- and Intermediate-Mass EMP Stars

AIP Conf. Proc. 990, 276 (2008); 10.1063/1.2905563

Abundance Ratios in Carbon-Enhanced Metal-Poor Stars and the Intermediate-Mass Star Initial Mass Function AIP Conf. Proc. 990, 197 (2008); 10.1063/1.2905540 


\title{
An Observational Perspective on Some Aspects of Early Stellar Nucleosynthesis
}

\author{
Christopher Sneden* and James E. Lawler ${ }^{\dagger}$ \\ ${ }^{*}$ Department of Astronomy and McDonald Observatory, University of Texas, Austin, TX 78731 \\ ${ }^{\dagger}$ Dept. of Physics, University of Wisconsin, Madison, WI 53706
}

\begin{abstract}
Some basic abundance results for low metallicity stars that were formed in the early days of the Milky Way Galaxy are summarized. Discussion is centered on two nucleosynthetic groups: the light $\alpha$ elements $(\mathrm{Mg}, \mathrm{Si}$, and $\mathrm{Ca}$ ), and the neutroncapture elements (those heavier than the Fe group, atomic numbers greater than 30). Emphasis is placed on the present state of stellar spectroscopic and atomic transition data.
\end{abstract}

Keywords: stellar abundances, low metallicity stars, nucleosynthesis

PACS: $26.30 .+\mathrm{k}, 95.30,95.75,95.85 . \mathrm{Kr}, 95.85 . \mathrm{Ls}, 97.10 . \mathrm{Cv}, 97.10 . \mathrm{Ex}, 97.10 . \mathrm{Tk}, 97.20 . \mathrm{Tr}, 98.35 . \mathrm{Bd}$

\section{INTRODUCTION}

The First Stars conference series focuses on "the physics of formation, life and death of the earliest stars and their impact on subsequent structure formation and chemical evolution of the Universe." ${ }^{1}$ Chemical compositions of low metallicity stars provide crucial input data in this area.

Today we know of stars spanning six orders of magnitude in overall metallicity, from supersolar $([\mathrm{Fe} / \mathrm{H}] \sim+0.5 \text {, e.g., }[1])^{2}$ to ultra-metal-poor $([\mathrm{Fe} / \mathrm{H}] \sim-5.5,[2])$. Several thousand metal-poor stars (arbitrarily defined as those with $[\mathrm{Fe} / \mathrm{H}]<-1$ ) have now been identified; most of these are members of the Galactic halo population. But it is worth remembering the pioneering paper [3] that announced identification of the first convincingly metal-poor stars HD 19445 and HD 140283. This paper appeared less than 60 years ago. It greatly underestimated the true metal deficiency of these stars, due to the novelty of the basic idea at that time. In fact, the paper states that, "The one possibly undesirable factor in our interpretation is the prediction of abnormally small amounts of $\mathrm{Ca}$ and $\mathrm{Fe}$... Although many workers have previously suspected the subdwarfs of having a low hydrogen content, it appears that, with respect to $\mathrm{Ca}$ and $\mathrm{Fe}$ at least, the subdwarfs [HD 19445 and HD 140283] are rich in hydrogen." Nearly a decade more was needed to reliably establish basic metallicity and abundance techniques and results for a small number of halo stars, and the field has grown steadily ever since.

\footnotetext{
${ }^{1} \mathrm{http}: / /$ www.lanl.gov/conferences/firststars3/

${ }^{2}$ We adopt the usual spectroscopic notations that for elements $A$ and $\mathrm{B}, \log \varepsilon \mathrm{A} \equiv \log _{10}\left(\mathrm{~N}_{\mathrm{A}} / \mathrm{N}_{\mathrm{H}}\right)+12.0$, and $[\mathrm{A} / \mathrm{B}] \equiv \log _{10}\left(\mathrm{~N}_{\mathrm{A}} / \mathrm{N}_{\mathrm{B}}\right)_{\star}$ $\log _{10}\left(\mathrm{~N}_{\mathrm{A}} / \mathrm{N}_{\mathrm{B}}\right)_{\odot}$. Also, metallicity is identified here with the stellar $[\mathrm{Fe} / \mathrm{H}]$ value.
}

From a nucleosynthetic perspective, the major groups in the Periodic Table heavier than helium are (a) Li-Be-B, whose very low abundances can be generated in a variety of circumstances, (b) the light " $\alpha$ " elements (even$Z, 6 \leq Z \leq 22$ ), (c) the light odd- $Z$ elements (of the same atomic number range), (d) the Fe-peak elements $(21 \leq Z \leq 30)$, and (e) the neutron-capture elements ( $n$-capture, $Z>30$ ). The qualitative and quantitative explosion of information on chemical compositions of low metallicity stars means that one short review is inadequate to do justice to the field. Here we choose to concentrate only on the $\alpha$ and $n$-capture elements, but many of the successes and remaining problems in determining their abundances over a large metallicity range can be applied to other element groups as well. Our discussion will not stray far from the observational realm; other papers in this volume will take up the theoretical issues.

\section{THE OBSERVABLE $\alpha$ ELEMENTS}

The most abundant elements heavier that helium in the Galaxy are those light elements whose dominant naturally occurring isotopes are composed of even multiples of $\alpha$ particles. These include $\mathrm{C}, \mathrm{O}, \mathrm{Ne}, \mathrm{Mg}, \mathrm{Si}, \mathrm{S}$, and $\mathrm{Ca}$. Unfortunately, of this list the noble gas Ne is spectroscopically inaccessible in cool star spectra; $\mathrm{C}$ and $\mathrm{O}$ can suffer abundance alterations through proton captures during various $\mathrm{H}$-burning cycles in stars of all types; and $\mathrm{S}$ has mostly weak transitions (and thus is difficult to detect at very low metallicities). This leaves only $\mathrm{Mg}, \mathrm{Si}$, and $\mathrm{Ca}$ as the practical representatives of the "pure" $\alpha$ element group for stars of all Galactic populations. 


\section{$\alpha$ Elements in "Ordinary" Metal-Poor Stars}

Pioneering early spectroscopic studies established that the $\alpha$ elements are relatively overabundant in metalpoor stars. The first large-sample investigation to show this was [4], which found that for G-dwarf stars with $[\mathrm{Fe} / \mathrm{H}] \leq-0.4$, the $\alpha / \mathrm{Fe}$ abundance ratio was larger than solar: $[<\mathrm{Mg}$, $\mathrm{Si}, \mathrm{Ca}>/ \mathrm{Fe}] \sim+0.2$, with some correlation of this ratio with space velocity as well. Subsequent studies over the past four decades have deepened our understanding of the $\alpha$-element abundance trends over the entire Galactic metallicity range $(+0.5>[\mathrm{Fe} / \mathrm{H}]>$ $-5)$ but have not fundamentally changed this qualitative conclusion. Spectroscopic surveys have also shown that $\mathrm{Ti}(\mathrm{Z}=22)$ is generally overabundant by amounts similar to $\mathrm{Mg}, \mathrm{Si}$, and $\mathrm{Ca}$. Thus it is often lumped together with the true $\alpha$ elements in nucleosynthesis discussions. But its dominant isotope is ${ }^{48} \mathrm{Ti}$, and thus Ti probably should not be included as an official member of the group.

The general $\alpha$-element enhancement is shared by virtually all low metallicity stars, both members of the general field and those in globular clusters. We illustrate this in Figure 1, which shows the run of $[\mathrm{Ca} / \mathrm{Fe}]$ as a function of $[\mathrm{Fe} / \mathrm{H}]$ for the metallicity regime relevant to globular clusters, $[\mathrm{Fe} / \mathrm{H}]>-2.6$. This figure is an updated version of Figure 4 in [5], to which we have added some new data on field thick disk stars and globular clusters, as cited in the figure caption.

A few conclusions can be drawn from Figure 1. First, within the observational uncertainties the average $[\mathrm{Ca} / \mathrm{Fe}]$ ratios of field and globular cluster stars are essentially indistinguishable for halo metallicities $([\mathrm{Fe} / \mathrm{H}]<$ $-1)$. Galactic chemical evolution models need to account for this general agreement. Either the elemental yields of high mass element donors (the sources for both $\mathrm{Ca}$ and Fe in the early Galaxy) in all environments are relatively simple functions of stellar metallicity, or the major nucleosynthesis events in the nascent Galaxy occurred prior to the condensation of globular clusters out of the original halo material. Second, $\mathrm{Ca}$ overabundances in globular clusters persist into the Galactic thin/thick disk metallicity domain $([\mathrm{Fe} / \mathrm{H}]>-1)$, while a decline to $[\mathrm{Ca} / \mathrm{Fe}] \simeq 0$ at $[\mathrm{Fe} / \mathrm{H}]=0$ is exhibited by field disk stars and open clusters alike. The highest metallicity globular clusters are disk residents, lying at low Galactic latitudes and often dust-obscured. It is not easy to obtain high resolution spectra of them suitable for full abundance analyses. Further exploration of the chemical compositions of clusters in this metallicity regime would be welcome.

Note the anomalously low $[\mathrm{Ca} / \mathrm{Fe}]$ ratios for $\mathrm{Pal} 12$ [16, 17], Rup 106 [16], and NGC 5694 [12] shown in

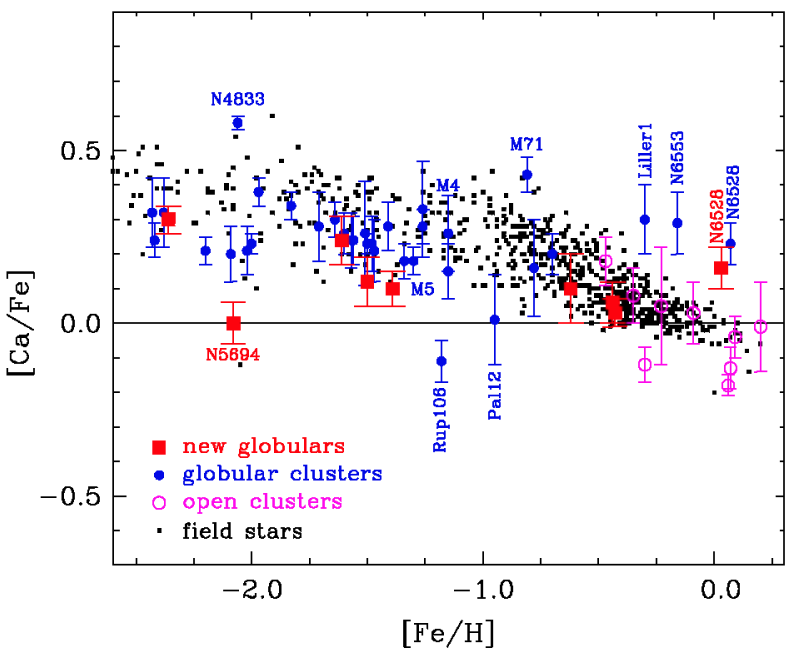

FIGURE 1. $[\mathrm{Ca} / \mathrm{Fe}]$ abundance ratios plotted against $[\mathrm{Fe} / \mathrm{H}]$ in the metallicity regime of Galactic globular clusters, $[\mathrm{Fe} / \mathrm{H}]>-2.6$. This figure is an updated version of Figure 4 in [5]. The points for "open clusters" (magenta open circles) "globular clusters" (blue filled squares) and most of the "field stars" (small black dots) are taken from [5], where citations to the original literatures sources can be found. New data on field thick disk stars (small black dots, not distinguished from the other field stars) are from [6], and new globular clusters (red squares) are from $[7,8,9,10,11,12,13,14]$. The new point for NGC 6528 from [13] is a renormalized abundance from [15], also shown in the figure.

Figure 1. These are three outer-halo clusters $[18]^{3}$ that are relatively far from the Galactic center. Some outer halo clusters, Pal 12 among them, are probably younger than most of the other globulars (see, e.g., [19]), and thus perhaps are not native to the Galaxy. Such objects may have been accreted later by the Galaxy, and thus their deviations from the mean $[\mathrm{Ca} / \mathrm{Fe}]$ values of other clusters may not require special explanation in Galactic chemical evolution. They may represent a fundamentally different population than the vast majority of globular clusters.

An intriguing trend of decreasing [Si/Ti] abundance ratios with Galactocentric distance has been identified by [20]. This trend has been attributed to the production of these abundances in different mass ranges of Type II supernovae that dominated element synthesis at different Galactocentric distances in the early Galaxy. The Galactic position influence does not appear to extend to $[\mathrm{Ca} / \mathrm{Fe}]$, as summarized in Figure 5 of [5].

\footnotetext{
3 The current version is available at http://physwww.mcmasterca/ harris/mwgc.dat
} 


\section{$\alpha$ Elements at Lowest Galactic Metallicities}

Much early Galactic nucleosynthesis effort in recent years has concentrated on stars with sub-globular metallicities, $[\mathrm{Fe} / \mathrm{H}]<-2.5$. It is expected that stars with lowest metallicities on average are the oldest, or at least were formed from early halo gas that was seeded with the fewest prior nucleosynthesis events. The first largesample abundance study of such stars was conducted by [21], who employed spectra of moderately high resolution, $\mathrm{R} \equiv \lambda / \Delta \lambda \simeq 22,000$, and modest signal-to-noise, typically $\mathrm{S} / \mathrm{N} \sim 35$. They discovered fundamental alterations in some abundance ratio trends with metallicity (particularly among the Fe-peak elements $\mathrm{Mn}, \mathrm{Cr}$, and Co), and increased star-to-star scatter of the ratios for many elements (especially in the $n$-capture domain). Recently, a series of papers by the "First Stars" group (e.g, $[22,23,24]$ and references therein) has employed much better $\mathrm{R}$ and $\mathrm{S} / \mathrm{N}$ spectra to refine and extend the [21] conclusions. In Figure 2 we show $\alpha$ abundance ratios $[\mathrm{Ca} / \mathrm{Fe}]$ and $[\mathrm{Si} / \mathrm{Fe}]$ from these two studies, and we have added data from $[25,26,6]$ for higher metallicity stars.

Taking up first the $[\mathrm{Ca} / \mathrm{Fe}]$ data in the top panel of Figure 2, the lowest metallicity stars on average continue the relatively constant $\sim 0.3$ dex overabundance value of the less extremely metal-poor stars that were considered previously. There is little to distinguish the results of [21] from those of [22], except in the few stars of each sample that deviate significantly from the mean $[\mathrm{Ca} / \mathrm{Fe}]$ value.

Inspection of the $[\mathrm{Si} / \mathrm{Fe}]$ data of Figure 2's bottom panel suggests a more interesting situation. At all metallicities the mean abundance ratio echoes that of of the top panel: $\langle[\mathrm{Si} / \mathrm{Fe}]\rangle \sim<[\mathrm{Ca} / \mathrm{Fe}]\rangle$. It is clear however that the star-to-star scatter is different: $\sigma[\mathrm{Si} / \mathrm{Fe}]>\sigma[\mathrm{Ca} / \mathrm{Fe}]$. If star-to-star $\alpha$ element abundance variations are real at very low metallicities, then Figure 2 contains direct evidence of prior individual nucleosynthesis events that would have seeded a very inhomogeneous early Galactic halo interstellar medium. The data of Figure 2 indicates that of the two surveys of stars with $[\mathrm{Fe} / \mathrm{H}]<-2.5$, the more recent study with better spectroscopic data has significantly reduced the star-to-star scatter. The total range in $[\mathrm{Si} / \mathrm{Fe}]$ is $\sim 0.6 \mathrm{dex}$ in $[22]$, in contrast to the $\sim 1.2 \mathrm{dex}$ spread reported in [21]

Below we consider whether much of the remaining star-to-star scatter in $[\mathrm{Si} / \mathrm{Fe}]$ values represent "observational" artifacts introduced at some point in the steps from telescopic data collection to derivation of abundances. First, we must stress that some low metallicity stars with genuinely anomalous $[\alpha / \mathrm{Fe}]$ ratios do exist in the Galactic halo. Several "low- $\alpha$ " examples have been found, beginning with the analysis of BD+80 245 by [27], which revealed a star with $[\mathrm{Fe} / \mathrm{H}] \simeq-2.1$ but $[\alpha / \mathrm{Fe}] \equiv<[\mathrm{Mg}, \mathrm{Si}, \mathrm{Ca} / \mathrm{Fe}]>\simeq-0.2[28]$, nearly 0.5 dex lower than the typical halo-star value. This star's
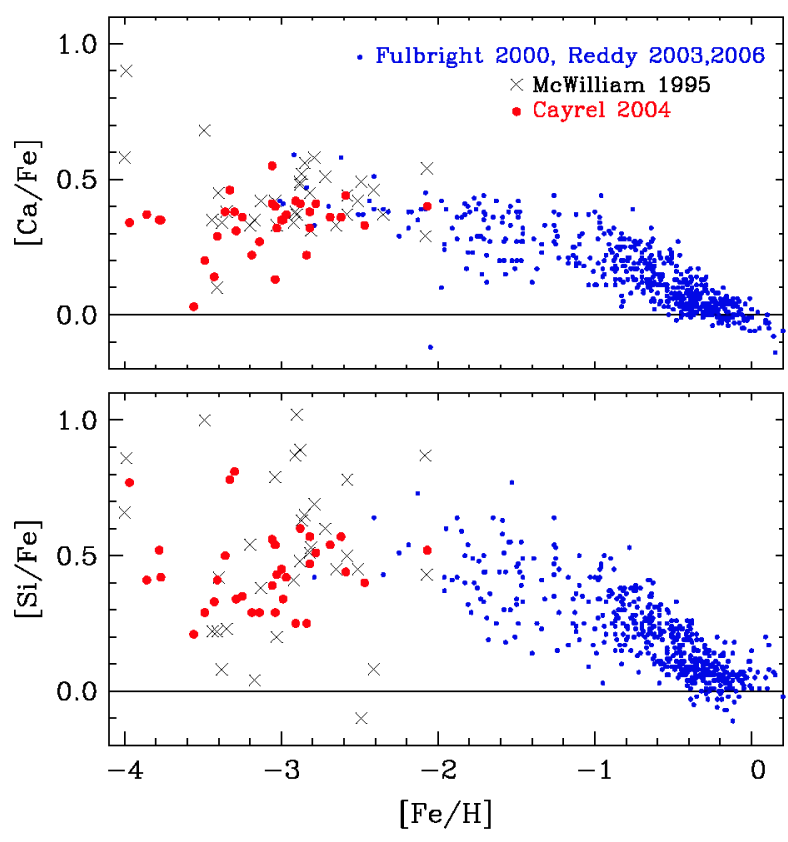

FIGURE 2. Abundance ratio trends for two $\alpha$ elements in the extended metallicity regime $0.0 \geq[\mathrm{Fe} / \mathrm{H}] \geq-4.1$, which includes most Galactic halo stars. The symbols are identified in the figure legend. The horizontal black lines at $[\mathrm{Ca} / \mathrm{Fe}]$ or $[\mathrm{Si} / \mathrm{Fe}]=0.0$ represent the solar abundance ratios of these elements. For this figure, data for lowest metallicity stars are taken from $[21,22]$, and we have filled in the rest of the metallicity range with data from $[25,26,6]$.

low- $\alpha$ general abundance distribution is seen in also in other serendipitous spectroscopic discoveries: G4-36 [28], CS 22966-043 [29, 28], and HE 1424-0241 [30]. This last star is noteworthy for its extremely low metallicity, $[\mathrm{Fe} / \mathrm{H}] \sim-4$, nearly 2 dex smaller than the other three stars in the list.

In Figure 3 we summarize the abundance pattern for $\mathrm{HE}$ 1424-0241 and $\mathrm{BD}+80245$. For this figure we follow [31] in plotting the differences of abundance ratios in these two stars to those of HD 122563, the wellknown bright very low metallicity giant star first discussed by [32] and repeatedly analyzed at high spectral resolution ever since. HD 122563 exhibits a "typical" halo-star abundance pattern, and in particular has $<[\mathrm{Mg}, \mathrm{Si}, \mathrm{Ca} / \mathrm{Fe}]>\simeq+0.36$ in its latest analy sis [31]. The abundance differences of the two low- $\alpha$ stars with respect to HD 122563 are similar but hardly identical: although on average both HE 1424-0241 and BD+80 245 are $\alpha$-poor compared to HD 122563, there are significant differences between them among the individual elements of interest here (e.g., $\mathrm{Mg}$ and $\mathrm{Si}$ ) as well as in other element groups (e.g., Co and $\mathrm{Ba}$ ).

The situation has been enriched through the discovery of a few very peculiarly $\alpha$-rich (or perhaps Fe-poor) low 


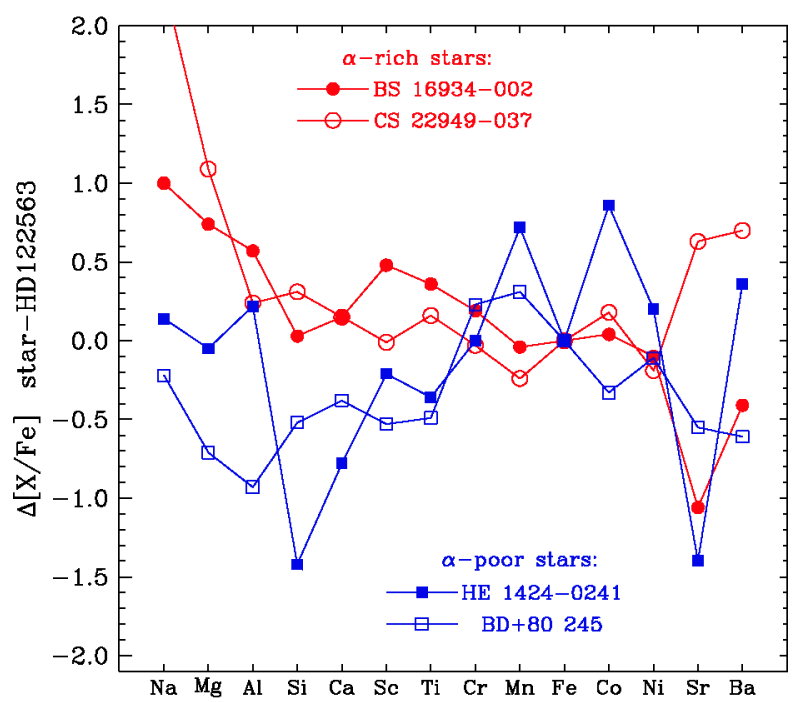

FIGURE 3. Abundances patterns of four halo stars with anomalous $[\alpha / \mathrm{Fe}]$ ratios compared to that of HD 122563, a metal-poor $([\mathrm{Fe} / \mathrm{H}] \simeq-2.7)$ giant with "normal" $\alpha$-element overabundances. Only elemental abundances derived for all of these stars are included in the figure. Reference to the abundance studies for these stars are given in the text.

metallicity stars, e.g., BS 16934-002 [31] and CS 22949$037[21,33]$. We also plot abundance differences of these two stars with respect to HD 122563 in Figure 3. The contrast between the $\alpha$-rich and $\alpha$-poor stars is $\sim 1$ dex or more in $[\mathrm{Mg}, \mathrm{Si}, \mathrm{Ca} / \mathrm{Fe}]$, well beyond any reasonable abundance uncertainties.

\section{Some Remaining Analytical Issues}

Unfortunately, there are some observational/analytical concerns attending light element abundance determinations in metal-poor stars must be addressed before nucleosynthetic interpretation can be done with confidence. To begin with one fairly extreme example, it has long been known that there are inconsistencies between abundances derived from different spectral features of the odd- $\mathrm{Z}$ element $\mathrm{Al}$. For lowest metallicity stars the only reliable $\mathrm{Al}$ abundance indicator is $\mathrm{Al}$ I $3961.54 \AA$ $(\chi=0.01 \mathrm{eV})$. At higher metallicities this line is very strong, and since it lies in the wing of the huge combined $\mathrm{Ca}$ II $\mathrm{H}$ and $\mathrm{H} \varepsilon$ spectral feature, observers usually switch to longer-wavelength lines such as the Al I 6696.03, 6698.67 A pair. The red and blue lines yield very discordant results, with the red lines suggesting that $[\mathrm{Al} / \mathrm{Fe}] \sim 0.0$ and the blue line consistently yielding $[\mathrm{Al} / \mathrm{Fe}]<-0.3$; see the discussions in, e.g., $[34,35]$ on causes and potential cures of this problem.

Abundance clashes from different spectral features of any element suggest first that a brief examination of basic transition probabilities used in the abundance analyses should be conducted. The $\log g f$ data for neutral-species lines of $\alpha$ elements $\mathrm{Mg}, \mathrm{Si}$, and $\mathrm{Ca}$, as well as for $\mathrm{Al}$, have improved dramatically in recent years. Spectra of these neutral species are sufficiently simple that theoretical results are preferred in most, but not all cases.

Consider first Mg I which is an alkaline earth spectrum. The presence of only two valence electrons outside of a Ne-like core with small relativistic effects leads to tractable calculations. Opacity Project (OP) results from [36] and Multi Configuration Hartree Fock (MCHF) results from $[37]^{4}$ are recommended by the NIST Atomic Spectra Database (NIST-ASD) ${ }^{5}$ for many of the important visible and near IR lines connecting low excited levels in Mg I. The main shortcoming of many of the OP results is the lack of a fine structure treatment. Individual transition probabilities are then based on the LS or Russell-Saunders approximation, which is usually satisfactory for strongly allowed lines but is sometimes in error for weaker lines. New experimental results from [38] have become available for the important (from an astrophysical viewpoint) $\mathrm{Mg}$ I $3 \mathrm{~s} 3 \mathrm{p}-3 \mathrm{~s} 4 \mathrm{~s}$ multiplet at 5167,5173 , and $5184 \AA$ (the Fraunhofer "b" lines). These new experimental results are based on the best modern method for determining transition probabilities. This experimental method combines lifetime measurements using laser induced fluorescence (LIF) and emission branching fraction measurements using a Fourier Transform Spectrometer (FTS). The agreement between the OP $\log g f$ values and the new experimental values for the $3 \mathrm{~s} 3 \mathrm{p}-3 \mathrm{~s} 4 \mathrm{~s}$ multiplet of $\mathrm{Mg} \mathrm{I}$ is $0.07 \mathrm{dex}$ or better. Although the uncertainty of the new experimental $\log g f$ values is $0.04 \mathrm{dex}$, it is clear that the NIST accuracy estimates are reasonable for the $\mathrm{OP}$ and $\mathrm{MCHF}$ results on $\mathrm{Mg}$ I.

The situation for Al I is similar. The NIST-ASD recommends primarily OP results [39]. Many of the important multiplets involve a single valence electron changing its orbit outside of a closed $3 \mathrm{~s}^{2}$ sub-shell. Longstanding difficulties, discussed above, in reconciling $\mathrm{Al}$ abundance values from the resonance $3 s^{2} 3 p-3 s^{2} 4 s$ multiplet at 3944 and $3962 \AA$ with abundance values from the red $3 s^{2} 4 s-3 s^{2} 5 p$ multiplet at 6696 and $6699 \AA$ are almost surely not due to problems with $\log g f$ values. The calculation of transition probabilities for the resonance multiplet is in many respects more difficult than for the red multiplets, but there have been multiple LIF measurements on the resonance multiplet (e.g. [40, 41, 42]). It is very likely that $\log g f$ values for the resonance multiplet

\footnotetext{
4 http://www.vuse.vanderbilt.edu/ $\sim$ cff $/$ mchf_collection $/$

${ }^{5}$ http://physics.nist.gov/PhysRefData/ASD/index.html
} 
of $\mathrm{Al}$ I are known to within 0.02 dex or better.

The addition of another $3 p$ valence electron makes $\mathrm{Si}$ I somewhat more difficult from a theoretical standpoint. Some additional theoretical and experimental work on $\log g f$ values for the most important multiplets of $\mathrm{Si} \mathrm{I}$ is desirable. The same can be said for $\mathrm{Ca}$ I. The available theoretical $\log g f$ values for the important multiplets of Si I and Ca I are not quite as good as those available for the important multiplets of Mg I and Al I. Happily, many yellow-red transitions of $\mathrm{Ca} I$ have been measured in the lab to high accuracy $( \pm 5 \%$, [43]). In short transition probability uncertainties of these species, with the possible exception of Si I, are unlikely to be the major sources of abundance uncertainties in the present analyses.

For Si I, next consider the features actually employed in the various studies. The surveys chosen for inclusion in Figure 3 were based on large stellar sample size; individual studies of single or a small handful of stars are not shown. But there is study-to-study heterogeneity in the spectroscopic features chosen for analysis. Specifically, the $[\mathrm{Si} / \mathrm{Fe}]$ abundances for the lowest metallicity stars have been determined almost exclusively from a single line: Si I 3905.53 A. In Figure 4 we show a partial Grotrian diagram for Si I, indicating the lower $(\chi=1.91 \mathrm{eV})$ and upper $(4.93 \mathrm{eV})$ levels of this transition. The figure also indicates the $4102.94 \AA$ line, a much weaker transition that arises from the same lower level as the $3905 \AA$ line. The $4102 \AA$ line has been used in a few stars. Higher excitation Si I lines are generally too weak to be detected at these metallicites. At larger values of $[\mathrm{Fe} / \mathrm{H}]$ these two lines become very strong, and their spectral regions are often too line-rich to yield reliable Si abundances. Therefore higher-metallicity surveys such as the ones included in Figure 2 [25, 26, 6] almost exclusively have used Si I lines in the yellow-red spectral regions. As examples we show the ones at $\mathbf{5 7 7 2 . 1 5}$ and $\mathbf{5 7 9 3 . 0 8} \AA$ in Figure 4; these transitions arise from the upper levels of the 3905 and $4102 \AA$ lines, respectively. Other yellow-red spectral lines are often used that have no direct coupling to the blue lines; see the cited studies for their particular $\mathrm{Si}$ I line choices. Rarely are red and blue Si I features studied in the same stars, and it is not obvious that the abundances from these different lines yield consistent results. There is almost no sample overlap in Figure 2 between the very metal-poor [21,22] and higher metallicity $[25,26,6]$ surveys.

A further concern on Si analyses has recently been identified: abundances determined exclusively from the Si I $3905 \AA$ line have a pronounced dependence on stellar effective temperature. This issue was discussed in [44], which presented abundance results for very metal-poor red horizontal branch stars spanning $5000 \mathrm{~K} \leq \mathrm{T}_{\text {eff }} \leq 6400 \mathrm{~K}$. In Figure 5 we show an extension of Figure 10 from [44], which shows a clear trend of

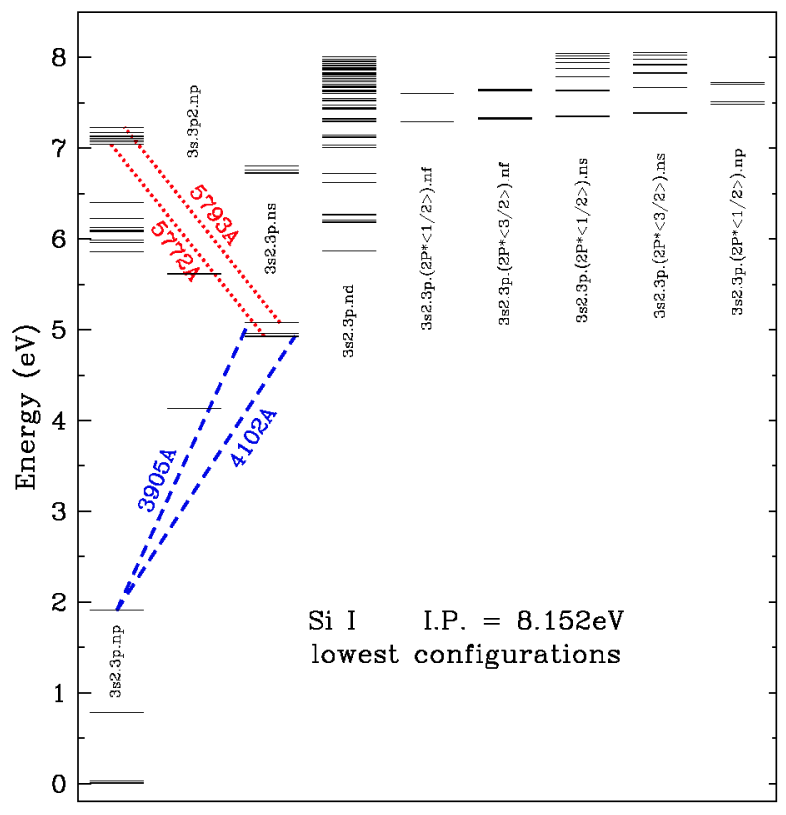

FIGURE 4. A partial Grotrian diagram for $\mathrm{Si}$ I. The first nine configurations are shown, along with four transitions that are commonly employed in Si abundance determinations in metalpoor stars. Data for this figure have been taken from the NISTASD web site.

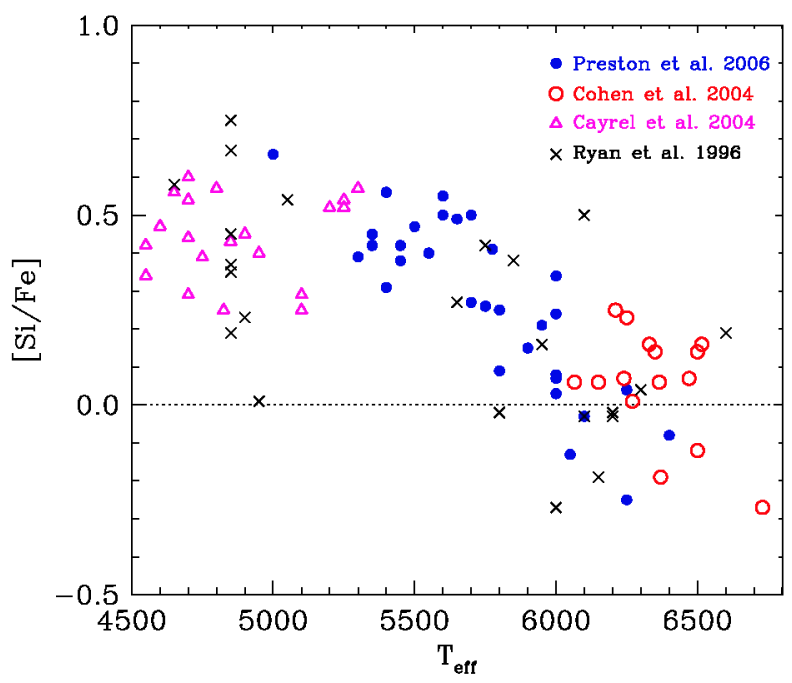

FIGURE 5. $[\mathrm{Si} / \mathrm{Fe}]$ relative abundances plotted as a function of stellar effective temperature. The data sources are given in the figure legend and cited in the text. The dotted horizontal line represents the solar $[\mathrm{Si} / \mathrm{Fe}]$ value.

[Si/Fe] ratios for the horizontal branch stars from $\sim+0.5$ at the cool end of the distribution to $\sim-0.1$ at the hot end. But these abundance ratios merge smoothly into the mean values for cooler metal-poor red giants [22] and warmer metal-poor stars near the main sequence turnoff 
[45]. Addition of abundances to Figure 5 from an earlier survey [35] that included stars of a larger temperature range reveals the same problem in those data as well (that study did call attention to the relatively large scatter in $[\mathrm{Si} / \mathrm{Fe}]$ values). The fundamental stellar atmosphere parameter dependence appears to be temperature alone, since among the warmer stars there is an admixture of similar-metallicities stars with very different gravities: $(1.8 \leq \log g \leq 2.8)$ and turnoff dwarf/subgiant stars $(3.6 \leq \log g \leq 4.6)$. The $3905 \AA$ line is contaminated with a $\mathrm{CH}$ molecular feature, but only in very $\mathrm{C}$-rich stars $[45,44]$ does the blending appear to be a serious issue for Si abundance analyses.

Further progress on the abundances of $\alpha$ and other light elements will come when all of them have at least been subjected to full statistical equilibrium (non-LTE) line formation computations. The astrophysical importance of $\mathrm{C}$ an $\mathrm{O}$ and the disagreements in their derived abundance from different atomic and molecular features has led to a successful effort in this area (e.g., [46, 47, 48] and references therein). Non-LTE studies of Ca I, Ca II [49] and Mg I, Mg II [50] have been recently reported. These are important studies and should be pursued for other relevant species.

To summarize the discussion of $\alpha$ elements, a very large number of studies over the past several decades has established that: (a) the mean $\alpha / \mathrm{Fe}$ at low metallicities is a factor of 2-3 larger than it is in the Sun; (b) significant trends of $\alpha / \mathrm{Fe}$ with metallicity cannot be definitely established for stars with $[\mathrm{Fe} / \mathrm{H}]<-1$; (c) a small number of very metal-poor stars substantially depart from the general $\alpha$ element abundance level; and (d) Si abundances (perhaps other elements as well) have analytical difficulties remaining to be sorted out. We began by defining $\mathrm{C}, \mathrm{O}, \mathrm{Ne}, \mathrm{Mg}, \mathrm{Si}, \mathrm{S}$, and $\mathrm{Ca}$ to be the official $\alpha$ elements, but quickly eliminated $\mathrm{C}, \mathrm{O}, \mathrm{Ne}$, and $\mathrm{S}$ (observational detection difficulties or susceptibility to alteration in H-burning cycles), and now have argued that a line formation problem or problems exist for $\mathrm{Si}$. What observed $\alpha$ abundances can we recommend with some confidence to stellar nucleosynthesis and Galactic chemical evolution modelers? At present $\mathrm{Ca}$, and perhaps $\mathrm{Mg}$. Clearly there is room for further work on all fronts in this area.

\section{THE OBSERVABLE NEUTRON-CAPTURE ELEMENTS}

The $n$-capture elements are those heavier than the Fepeak, normally defined as $Z>30$. These elements often are comprised of many isotopes, the vast majority of which are built in either the $s$-process or the $r$-process. These $n$-capture processes both involve neutron additions to target seed nuclei (such as those of the Fe-peak) followed by $\beta$ decays of any unstable resultant (neutronrich) nuclei. The $s$ - and $r$-processes arise in different $n$ capture/ $\beta$-decay regimes. If the neutron fluxes are small enough that $\beta$ decays of unstable nuclei can adjust between successive neutron additions, then element synthesis "slowly" proceeds up the valley of $\beta$-stability; this is the $s$-process. If however neutron capture rates overwhelm $\beta$-decay rates, extremely neutron-rich nuclei are "rapidly" created, which then decay back to $\beta$-stability; this is the $r$-process. Various isotopes of $n$-capture elements can be created by the $r$ - or the $s$-process, or by both ${ }^{6}$

\section{$r$-Process or $s$-Process Element?}

The concept of an $r$ - or $s$-process element is formally meaningless for all but a few $n$-capture elements. Those in the atomic number range $Z=84-89$ are composed of isotopes that are unstable very short timescales, and thus the $s$-process cannot create elements beyond Bi $(Z=83)$. All elements heavier than this are thus truly $r$-process ones, but of course only Th and $U(Z=90,92)$ survive on cosmic timescales to be detectable in stellar spectra.

For other $n$-capture elements the $r$ - and $s$-process fractions can be determined at the isotopic level only through analyses of solar system meteoritic material. Fortunately, reactions involved in the $s$-process can be studied in the lab. With these data, the $s$-process fraction of each isotope can be computed empirically $[51,52,53]$. The broad outlines of $r$ - and $s$-process contributions to each element have been well understood since the pioneering effort of [54]. To illustrate this, in Figure 6 we plot the $r$-only and $s$-only elemental abundances ${ }^{7}$ for solar system material from [51] and from [53]. As the meteoritic abundance and $s$-process reaction data have improved so has the accuracy of $r-/ s$-process fractional breakdown. But the original estimates of [51] have proven to be remarkably robust.

Overplotting of $r$ - and $s$-process abundances in Figure 6 shows that each of these $n$-capture mechanisms contributes about half of the solar-system content of $Z>30$ elements. However, there are pronounced variations among individual elements: $\mathrm{Sr}, \mathrm{Ba}, \mathrm{La}$ are predom-

\footnotetext{
6 Many $n$-capture elements also have some relatively proton-rich isotopes, but these are very minor abundance constituents and their presence is essentially undetectable in stellar spectra.

7 The elemental abundances are determined by summing the individual isotopic $r$-only and $s$-only abundances. The data for Figure 6 begin with published values that are relative number densities on the meteoritic scale, that is normalized to $\mathrm{N}_{\mathrm{Si}} \equiv 10^{6}$. Conversion to the astronomical scale $\varepsilon_{\mathrm{H}} \equiv 10^{12}$ was accomplished by multiplying the meteoritic number densities by $10^{1.55}$
} 


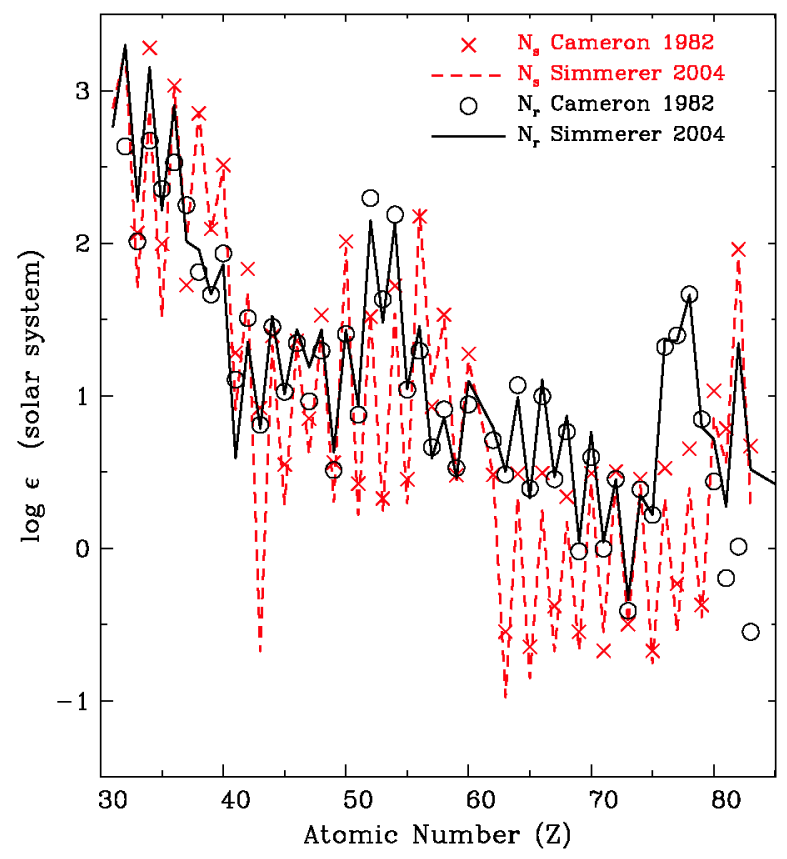

FIGURE 6. The $n$-capture elemental abundances of solarsystem (mostly meteoritic) material, separated into the pure $r$-process and $s$-process components. Lines and symbols are defined in the figure legend. Comparisons between observed abundances of low metallicity $r$-process-rich stars and the solar-system $r$-process-only distribution are thus differences between the observed total stellar abundances and (a scaled version of) the black line of this figure.

inantly products of the s-process, and $\mathrm{Eu}, \mathrm{Gd}$, and $\mathrm{Dy}$ and are nearly pure $r$-process products. Thus these and others are referred to as $r$ - or $s$-process elements. It must be emphasized that this casual division applies only to solar-system material.

\section{Neutron-Capture Elements at Low Metallicity: $r$-Process Stars.}

The $n$-capture abundance distributions of many metalpoor stars stand in sharp contrast to that of the solar system. The pioneering survey of some brighter metal-poor (mostly) giant stars by [59] clearly showed examples of $[\mathrm{Ba} / \mathrm{Eu}] \ll 0$ (see their Figures 4 and 5). This was interpreted [60] as evidence for production of $n$-capture elements solely by the $r$-process prior to the formation of these stars. Isolated examples of somewhat $r$-processrich or $s$-process-poor stars have been discussed in the literature (e.g., [61, 62, 63, 64]), but the first convincing example of an extremely $r$-process-rich star was CS 22892052 , serendipitously discovered in the [21] survey. This object has been subjected to a number of investigations with increasing quality of spectroscopic data and atomic physics input $[65,66,67,68,56,69]$. All of these studies agree on the general abundance properties: $[\mathrm{Fe} / \mathrm{H}] \simeq-$ $3.1,[\mathrm{Eu} / \mathrm{Fe}] \simeq+1.6,[\mathrm{Eu} / \mathrm{Ba}] \simeq+0.9$. The abundances of elements with $Z \geq 56$ fit very well a scaled solarsystem $r$-process-only abundance distribution (the solid black line in Figure 6).

The solar-system $r$-process abundance pattern for the rare earth and heavier elements is repeated nearly exactly in all metal-poor stars that are generally enriched in $n$ capture elements and have $[\mathrm{Ba} / \mathrm{Eu}]>+0.5$, e.g., $[57,70$, $58,69,55]$. This is illustrated in Figure 7, which is taken from [55]; their Figure 11). For $Z>56$ the deviations for most stars are well within the uncertainties of the various studies.

The excellent star-to-star abundance agreement for nearly all rare earths stems from the ability to observe large numbers of ionized transitions in $r$-process-rich stars. First ionization potentials of the rare earths are $\sim 6 \mathrm{eV}$, and therefore these elements are completely ionized in the stars of interest. The lowest energy levels of the ions are well populated, and transitions from these levels dominate the spectra.

The atomic structures of rare-earth ions are very complex. Figure 8 includes partial Grotrian diagrams for two examples: Sm II and Gd II. Open f-shells of these species lead to extremely rich spectra. The spectra are so rich that boxes or blocks are often used in Grotrian diagrams such as Fig 8 to represent the 10's to 100 's of levels in a subconfiguration. The overlap of low configurations, visible in this figure, leads to significant configuration interaction. Russell-Saunders or LS coupling breaks down to a substantial extent this deep into the periodic table due to relativistic effects that overwhelm Coulomb repulsion in the Hamiltonian. The theorist is faced with an open fshell, the need to include 1000's of interacting levels, a breakdown of LS coupling, and other strong relativistic effects. Although the determination of accurate $\log g f$ values for the rare earths is a formidable theoretical challenge, the experimental method of combining radiative lifetimes from LIF with branching fractions from an FTS has proven to be both accurate and efficient. Recent work on Gd II and Sm II [72, 71], from which Figure 8 was constructed, are good examples. Reliable $\log g f$ values for important transitions of many other rare-earth ions and atoms are now available from combining LIF and FTS measurements (see the references in the introductory paragraph of [71]).

\section{Isotopes in $r$-Process-Rich Stars}

Several $Z>56$ elements have transitions whose hyperfine and isotopic substructures act to broaden their absorption profiles beyond those created by the stellar at- 


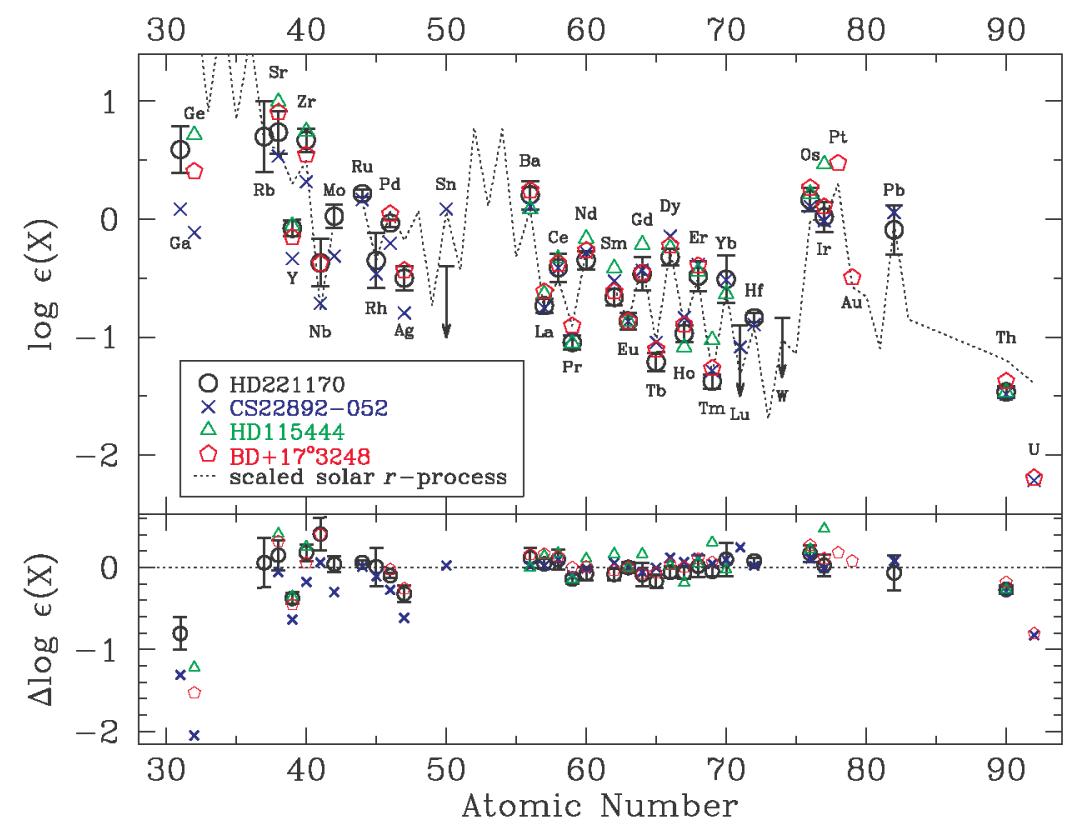

FIGURE 7. Comparison of $n$-capture abundance of several $r$-process-rich stars (HD 221170 [55], CS 22892-052 [56], HD 115444 [57], and $\mathrm{BD}+173248$ [58]) with each other and with the solar-system abundance distribution [53]. This figure is a reproduction of Figure 11 of [55]. In the top panel all abundances have been normalized to the the observed Eu abundance of HD 221170 . In the bottom panel is a difference plot of the stellar and solar-system abundances, again normalizing to the Eu abundances. The points for $U$ should be considered as upper limits.

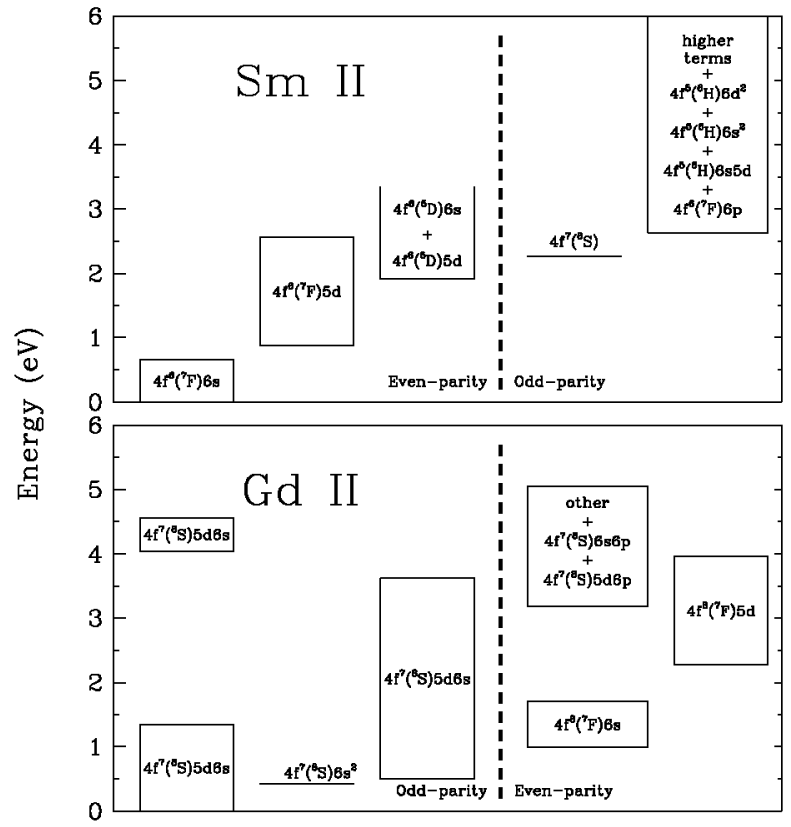

FIGURE 8. Schematic partial Grotrian diagrams for Sm II and Gd II. The top and bottom panels are redrawn Figure 1 of [71] and Figure 1 of [72], respectively. Each rectangle indicates the energy range of a large number of sublevels of the named configuration. The rectangle that is open at the top for Sm II indicates that energy level assignments are not complete for those configurations. mosphere thermal plus turbulent motion combined with the spectroscopic instrumental smearing. The largest effect of this sort occurs for Eu, which has two naturallyoccurring isotopes with a solar-system meteoritic abundance ratio $\mathrm{N}\left({ }^{151} \mathrm{Eu}\right) / \mathrm{N}\left({ }^{153} \mathrm{Eu}\right)=0.478 / 0.522=0.916$ (e.g., [73] and references therein). Studies of the solar photospheric spectrum $([74,75]$ and $r$-process-rich spectra $([76,77]$ yield isotopic ratios in accord with this value, i.e. $\mathrm{N}\left({ }^{151} \mathrm{Eu}\right) / \mathrm{N}\left({ }^{153} \mathrm{Eu}\right) \simeq 1.0$. Most of the other odd-Z rare earths ( $\mathrm{La}, \mathrm{Pr}, \mathrm{Tb}, \mathrm{Ho}, \mathrm{Tm}, \mathrm{Lu}$ ) have wide hyperfine structures easily detectable in their observable transitions. But each of these elements has just one naturally-occurring isotope, rendering isotopic discussion moot.

The even- $Z$ rare earths have as many as seven naturally-occurring isotopes. Their isotopic abundance mixes can be substantially different in $r$ - and $s$-process production environments. However, detection of these isotopes is challenging, since the isotopic wavelength splitting is usually smaller than the stellar/instrumental line broadening. Most attempts to assess isotopic fractions have concentrated on the Ba II $4554.04 \AA$ transition. Hyperfine substructure exists for odd-A isotopes ${ }^{135,137} \mathrm{Ba}$ but not for even- ${ }^{134,136,138} \mathrm{Ba}$. Thus the intrinsic Ba II $4554 \AA$ line profiles produced by the odd-Z isotopes will be broader in wavelength than will those of the even- $Z$ isotopes. An $r$-process synthesis yields a 
higher fraction of odd- $Z$ isotopes than does an $s$-process one. Although the Ba line is often very strong and saturated, making analysis complicated, authors have been able to assess the odd- $Z$ ( $r$-process-rich) contribution to Ba through estimates of $\mathrm{N}\left({ }^{135,137} \mathrm{Ba}\right) / \mathrm{N}\left(\mathrm{Ba}_{\text {total }}\right)$, e.g., $[78,79]$. In the first large-sample survey in this area [80], increasing values of $\mathrm{Ba}$ isotopic fractions (that is, increasing $r$-process contributions to $\mathrm{Ba}$ ) positively correlate with $r$-process basic elemental abundance indicator [Eu/Ba]. Finally, attempts are being made to extend the rare-earth isotopic work to $\mathrm{Sm}$ and $\mathrm{Nd}$ $[81,82]$.

\section{Thorium and Uranium}

The observed abundances for Th and upper limits for $\mathrm{U}$ seen in Figure 7 lie substantially below their solarsystem values. This is most likely due to their longlived radioactive decay, and gives rise to hopes that these elements in $r$-process-rich low metallicity stars could act as chronometers, and the age of the $n$-capture material could be estimated from the observed "deficiencies" of Th and/or U compared to stable $n$-capture elements such as those illustrated in Figure 7,

Attempts to employ Th in cosmochronometry began with discussion of $\mathrm{Th} / \mathrm{Nd}$ ratios in disk stars by [83]. However, since the Nd abundance of solar-system material is due largely to the $s$-process (Figure 6: $\mathrm{N}_{r} / \mathrm{N}_{s}=$ $0.352 / 0.484=0.73$ [53]), the interpretation of observed $\mathrm{Th} / \mathrm{Nd}$ ratios is complex. The general idea was refined by [84], who employed $\mathrm{Th} / \mathrm{Eu}$ ratios because $\mathrm{Eu}$ in most stellar abundance mixes is nearly a pure $r$-process product $\left(\mathrm{N}_{r} / \mathrm{N}_{s}=0.090 / 0.003=30\right)$. Application to the Th II detections in CS 22892-052 have yielded "ages" of $13 \mathrm{Gyr}$ with uncertainties of $\sim 3 \mathrm{Gyr}$ (e.g. $[85,86]$ ), consistent with other kinds of age determinations for our Galaxy.

Unfortunately $\mathrm{Th} / \mathrm{Eu}$ cosmochronometry depends to a large extent on the assumed production ratio of these elements, and therefore concerns have been raised (e.g. [87]) on the reliability of $\mathrm{Th} / \mathrm{Eu}$ age estimates. This issue has been highlighted on the observational front by the detection of $U$ in the very $r$-process-rich star CS 31082-001 [70]. This star also has a very large relative abundance of Th, such that a simple implied age based on Th/Eu is very young, inconsistent with other properties (e.g., very low metallicity) of the star. The abundance ratio $\mathrm{Th} / \mathrm{U}$ in CS 31082-001 however yields an age of $\simeq 14$ Gyr for the star's $n$-capture material (e.g, [88]). This suggests that the supernova(e) that created the actinide elements for the star may have been in general "boosted" their production level above that estimated to have occurred prior to the creation of the solar system. A few other stars with

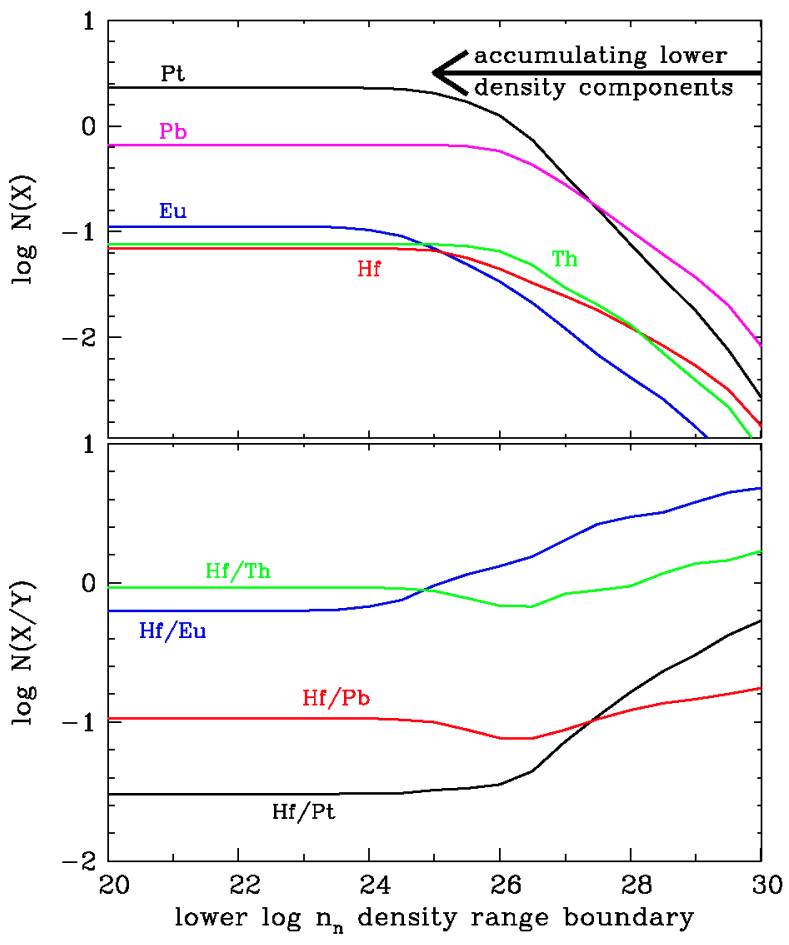

FIGURE 9. Reproduction of Figure 12 of [91], showing the predicted $r$-process abundances (top panel) and their ratios (bottom panel) for various neutron (high) density ranges for the heavy $n$-capture elements $\mathrm{Eu}, \mathrm{Fh}, \mathrm{Pt}$, and $\mathrm{Pb}$. See that paper for details of the computations. The abscissa represents the addition of individual $r$-process neutron density components, beginning at highest densities $\left(\log n_{n}=30\right)$ and accumulating lower-density components from right to left.

possible actinide boosts have been identified by [69]. On the other hand, a second solid U detection in $r$-processrich low metallicity material has now been announced for the star HE 1523-0901 by [89]. All of its abundance ratios involving Th and $U$ yield implied ages of $\simeq 13 \mathrm{Gyr}$.

The actinide cosmochronometry issue will not be completely solved until enough stars with solid Th and $U$ detections are studied in detail. In this work, the survey of [90] to identify new $r$-process-rich stars (as well as other surveys in various stages of completion) is very important. Followup careful investigation of all stars from this study estimated to have $[\mathrm{Eu} / \mathrm{Fe}]>+1$ and $[\mathrm{Ba} / \mathrm{Eu}]<0$ is recommended. Unfortunately, nearly all Th II and U II spectral features are weak, blended, or both. Analyses of these lines must always be done with care. 


\section{Indirect Observational Approaches to Actinide Production Predictions}

It is easy to understand why large uncertainties might exist in Th/Eu or U/Eu production ratios: the atomic number difference between Eu and the actinides is nearly 30, and extrapolations from Eu production are not easy to make. Some progress can be had by considering the abundance ratios involving Eu and the heaviest stable elements. These are the so-called $3^{r d} r$-process peak elements, of which the observable members are Os, Ir, Pt, and $\mathrm{Pb}(\mathrm{Z}=76-78,82){ }^{8}$ The Os-Ir-Pt group has been investigated in a sample of 11 metal-poor $r$-process-rich star by [92], who suggest that abundances of these elements strongly correlate with those of the rare earths, e.g. $[<\mathrm{Os}, \mathrm{Ir}, \mathrm{Pt}\rangle / \mathrm{Eu}] \simeq 0$. This supports the argument that a single abundance distribution is shared by solar-system and low metallicity $r$-process elements (see Figure 7).

Unfortunately, the detectable spectral features of the $3^{\text {rd }} r$-process peak elements are just a few intrinsically weak transitions of the neutral species that mostly occur in the UV spectral domain $(\lambda<4000 \AA)$. Happily, the lab transition data for the lines of interest in this effort appear to be in good shape $[93,94,95]$. But the observational uncertainties for the [Os,Ir,Pt, $\mathrm{Pb} / \mathrm{Eu}]$ are large, beginning with stellar model atmosphere uncertainties inherent to the comparison of neutral-species transitions with the ionized-species ones that comprise the only detectable features of the rare earths and the actinides.

The heaviest element that presents ionized lines in the spectra of metal-poor stars is $\mathrm{Hf}(Z=72$, just beyond the rare earth group). Its $r$-process potential importance has been indicated by recent calculations of [91], who suggest that the production of $\mathrm{Hf}, \mathrm{Pt}, \mathrm{Pb}$, and $\mathrm{Th}$ are linked. We illustrate this in Figure 9 (Figure 12 of [91]). The probable neutron density range that can reproduce solarsystem and $r$-process-rich stellar abundance distributions is $23 \leq \log n_{n} \leq 28$. Within those neutron density limits, the production ratios $\mathrm{Hf} / \mathrm{Eu}$ and $\mathrm{Hf} / \mathrm{Th}$ are nearly constant. Thus Hf could act as an $r$-process bridge element between $\mathrm{Eu}$ to $\mathrm{Th}$.

Recent lab studies $[96,97]$ have provided accurate new transition data for $\mathrm{Hf}$ II lines, and [97] have applied these data to high resolution spectra of $10 r$-processrich metal-poor stars. A brief summary of that abundance study is given in Figure 10. The top panel gives La/Eu ratios as a function of metallicity. These abundances suggest that the observed $\mathrm{La} / \mathrm{Eu}$ values and those predicted for the $r$-process are essentially the same; all the stars deserve to be labeled as $r$-process-rich. In the bottom panel

\footnotetext{
8 Au I has strong resonance lines but they lie in the deep-UV spectral region, and $\mathrm{Bi} \mathrm{I}$ hs only one very weak transition near $3000 \AA$.
}
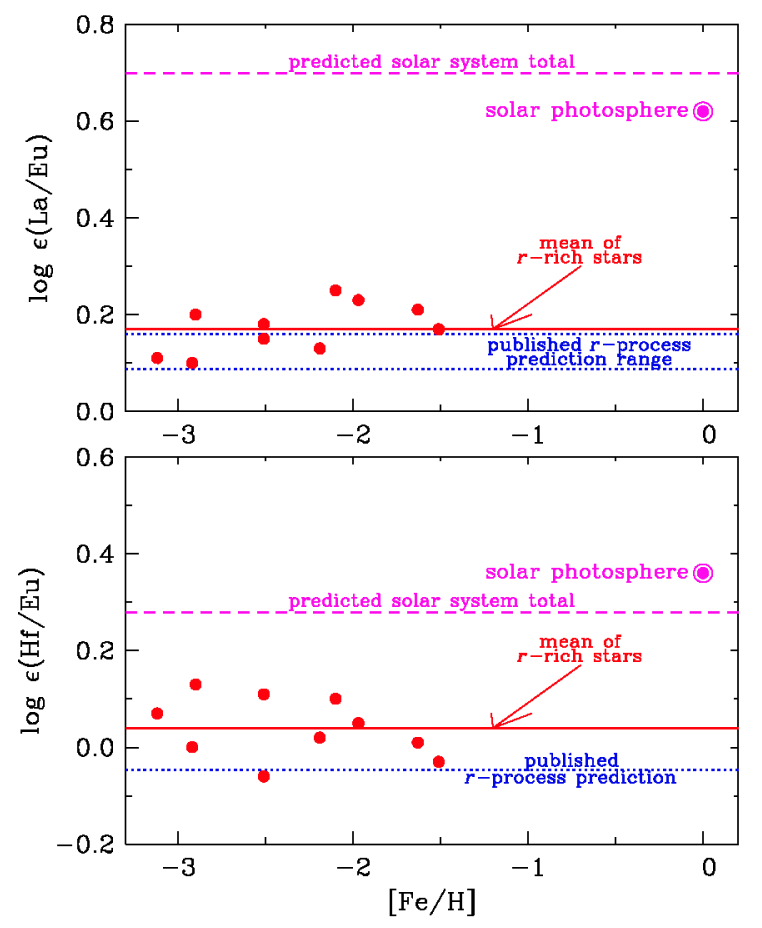

FIGURE 10. Correlation of $\mathrm{La} / \mathrm{Eu}$ and $\mathrm{Hf} / \mathrm{Eu}$ abundance ratios in $r$-process-rich low metallicity stars. This figure is derived from Figures 6 and 7 of [97], which should be consulted for detailed descriptions of the horizontal lines.

of Figure $10 \mathrm{Hf} / \mathrm{Eu}$ ratios are presented. Some details of observed and predicted values deserve further study, such as the offset between the predicted solar-system total ( $r$-process plus $s$-process) and solar photospheric abundance, and the $\simeq 0.1$ dex average overabundance of the metal-poor star points compared to expectations. But the simple message conveyed in these data is that $[\mathrm{Hf} / \mathrm{Eu}]$ is a constant abundance ratio in $r$-process synthesis environments, as suggested by the computations of Figure 9. Clearly a large-sample survey of Eu, Hf, and Th abundances in these kinds of stars is warranted.

\section{The Lighter $n$-Capture Elements in $r$-Process-Rich Stars}

Returning to Figure 7, inspection of the atomic number domain $Z<56$ suggests more that more star-tostar scatter exists for these elements than their heavier $r$ process counterparts. Perhaps more interesting, however, is to consider the variation in the bulk abundances of the lighter to the heavier elements. It has been known for some time that ratios such as $(\mathrm{Sr}, \mathrm{Y}, \mathrm{Zr}) /(\mathrm{Ba}, \mathrm{La}, \mathrm{Eu})$ have large variations among low metallicity stars. Two recent studies $[98,99]$ are noteworthy in demonstrating that 
on average the light-to-heavy $n$-capture abundance ratio (defined in several complementary ways) increases substantially as overall metallicity decreases. This is more attributable to a fall-off in the level of the heavier elements than an increased production of the lighter ones. Indeed, [92] found that the abundance of Ge (one of the very lightest of the official $n$-capture elements) was very well correlated with the abundance of Fe in their 11-star sample, and nearly uncorrelated with abundances of the traditional $r$-process elements such as Eu.

Theoretical interpretation of this issue is beyond the scope of this paper. There are suggestions of at least two different $r$-process sites/mechanisms (beginning with [100]), production in essentially a single $r$-process but varying neutron density domains (e.g., [91]), or an asyet not understood additional $n$-capture process [101]. Instead, we limit our comments to some observational issues.

First, the abundances of $\mathrm{Sr}, \mathrm{Y}$, and $\mathrm{Zr}(\mathrm{Z}=38-40)$ are determined from multiple lines with well-determined transition probabilities, $[102,103,104,105]$ arising from low-excitation levels of ionized species. Their abundances should be reliable, and ratios of these elemental abundances to those of the rare-earth group should have relatively small uncertainties. Caution should be used when only Sr abundances represent these elements, as in that case usually only the very strong $4077,4215 \AA$ resonance lines are analyzed.

More problematic are the elements in the $Z=41-48$ atomic number range. Nearly all of their useful transitions in metal-poor stars lie in the complex spectral regions below $4000 \AA$. Only a few usually very weak lines represent each element. Only $\mathrm{Nb}(Z=42)$ has detectable ionized-species transitions. The largest number of abundances in this element group have been reported for $\mathrm{Ag}$ $(Z=47)$. This element is represented in stellar spectra only by its resonance doublet at 3280 and $3382 \AA$; both of these lines are blended and usually weak. Caution should be exercised in interpretation of all elemental abundances in this atomic number regime.

\section{Neutron-Capture Elements at Low Metallicity: $s$-Process Stars}

Many metal-poor stars possess enormous enhancements of $\mathrm{C}$ and (usually) $n$-capture elements synthesized in the $s$-process. In general, these stars are more easily identified than those with $r$-process enhancements, because the CH molecular "G-band" stretches over 4200$4400 \AA$ in wavelength. Anomalously strong G-bands are thus easily spotted in low resolution spectroscopic surveys of halo stars. The number of C-rich stars appears to increase with decreasing metallicity; different authors
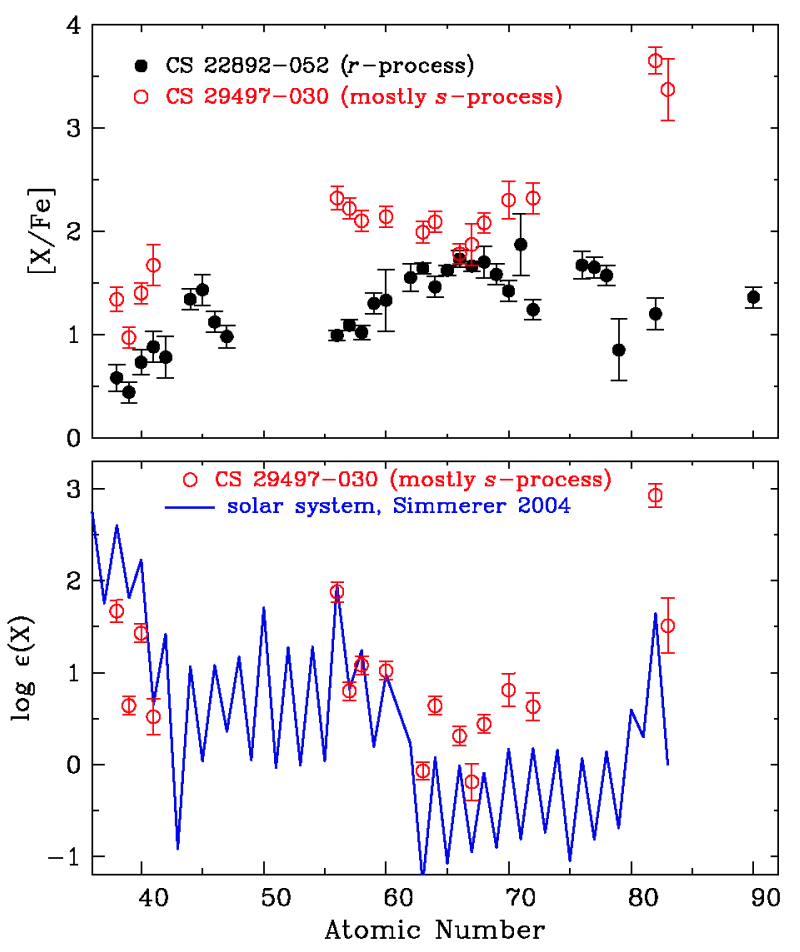

FIGURE 11. Top panel: comparative relative abundance enhancement patterns $[\mathrm{X} / \mathrm{Fe}]$ of two well-studied $n$-capture-rich stars. The CS 22892-52 data are from [56] while those of CS 29497-030 are from [106]. Bottom panel: comparison of the abundances of CS 29497-030 on the "absolute" $\log \varepsilon(\mathrm{X})$ scale with the solar-system $s$-only distribution ([53]). The solar abundances have been shifted by -0.25 dex to force agreement of stellar and solar values for $\mathrm{Ba}$.

$[107,108]$ suggest figures in the range of $\sim 10-25 \%$ of the total number of stars with $[\mathrm{Fe} / \mathrm{H}]<-2$.

The metal-poor stars with $\mathrm{C}$ and $s$-process overabundances have been studied extensively at high spectral resolution. The $s$-process-rich stars are easily distinguished from the $r$-process-rich ones even in the absence of $\mathrm{C}$ abundance information. This is illustrated in the top panel of Figure 11, in which examples of both $n$-capture abundance enrichments $[\mathrm{X} / \mathrm{Fe}]$ are shown. The contrast between the abundances of CS 22892-052 ( $r$-processrich) and CS 29497-030 ( $s$-process-rich) would even be more sharply delineated if the latter star had a more "pure" $s$-process signature. But detailed examination of in particular the rare-earth abundances in such stars (e.g., $[106,120])$ suggests that many of the $s$-process-rich stars also have underlying weaker $r$-process overabundances.

Additionally, the $s$-process abundance pattern in low metallicity stars is substantially different than that of metal-rich ones. To illustrate this we compare the actual abundances of CS 29497-030 in $\log \varepsilon$ units to the solar-system $s$-process abundances. Normalizing the two data sets at the CS 29497-030 Ba abundance, it is clear 


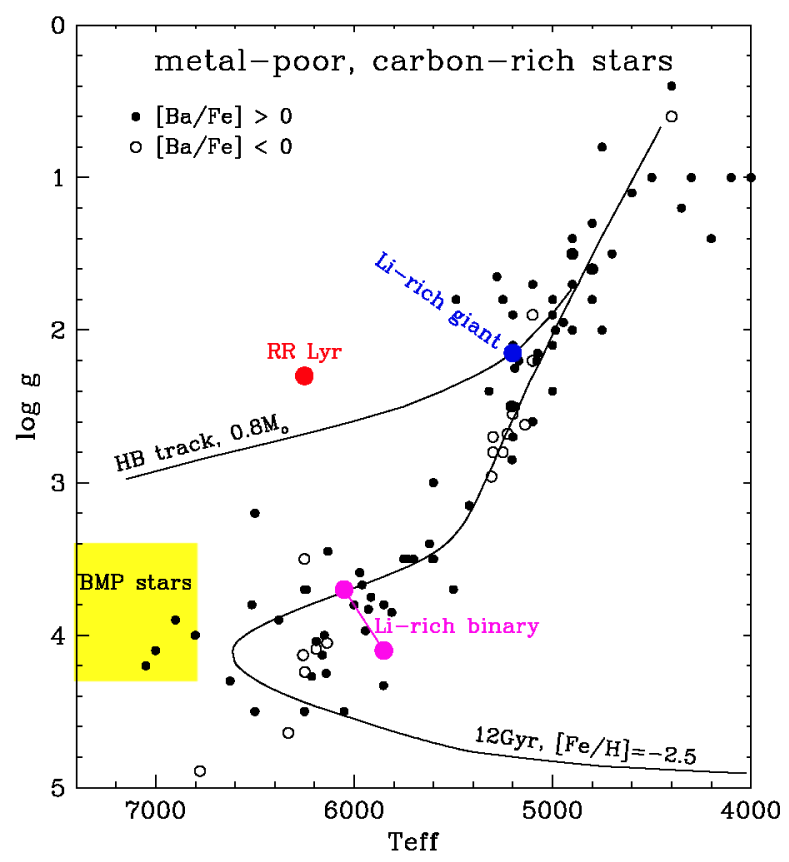

FIGURE 12. A ( $\left.\mathrm{T}_{e f f}, \log g\right) \mathrm{HR}$ diagram for metal-poor Crich stars. The stars are differentiated by filled/open circles according to their $s$-process enhancement, as indicated in the figure legend. Data have been taken from a number of sources: $[109,110,111,112,113,114,33,115,116][117,45,118,90$, $119,120,121,122],[80,23,123]$ The yellow rectangle denotes the approximate domain of the blue metal-poor (BMP) stars. The BMP star CS 29497-030 [106] is included in the plot but not given a special symbol. Individual stars with unusual characteristics are displayed with special symbols: The RR Lyr star TY Gru [124], red dot; the near main-sequence turnoff binary CS 22964-161 [125], magenta connected dots; and HB/RGB Li-rich star HK II 17435 [126], blue dot.

the metal-poor case is relatively deficient in the lighter elements Sr-Y-Zr and very enhanced in the heaviest elements $\mathrm{Pb}$ and $\mathrm{Bi}$. The overabundances of $\mathrm{Pb}$ are so large that this and many similar stars are labeled "lead stars. For example, in spite of the low metallicity of CS 29497 $030,[\mathrm{Fe} / \mathrm{H}] \sim-2.5$, its $[\mathrm{Pb} / \mathrm{Fe}] \sim+3.7$ translates to $[\mathrm{Pb} / \mathrm{H}] \sim+1.2$, about 10 times more lead than in the Sun.

It has long been established $[127,128,129]$ that metalrich and mildly metal-poor C- and s-rich stars are predominantly binaries with undetectable companions. We now know that the same is true of the very metal-poor ones as well, e.g., [29, 130]. The orbital periods ty pically are hundreds to thousands of days, but the eccentricities are fairly small, e.g., [29]. The evolutionary scenario that created the low metallicity $\mathrm{C}$ - and $s$-process-rich stars seems clear. The original primary star of these systems was a low-intermediate mass star that reached the asymptotic giant branch (AGB) stage late in its life, experienced the so-called $3^{\text {rd }}$ dredgeup phase in which its He-burning products ( $\mathrm{C}$ directly and $s$-process elements indirectly) to its surface, and ejected these new elements into its surroundings, where they were picked up by the lower mass star. The primary became a now-invisible white dwarf, and we see its companion today

What about the C-rich low metallicity stars that do not exhibit enhanced $s$-process abundances? The evolutionary status of these stars is not certain. Based on a survey of $26 \mathrm{C}$-rich stars [123] the authors conclude that, "No well-established model exists to explain the Ba-normal CEMP [carbon-enhanced metal-poor] stars, although Ryan et al. (2005) [[131]] suggested that they form from carbon-rich gas (from a massive progenitor star population) in contrast to the AGB progenitor paradigm, which explains many features of Baenhanced objects." The earlier suggestion was based on the tendency of $s$-process-weak C-rich stars to be giants, whereas the $s$-process-rich stars occur at all evolutionary stages. Now that the sample of C-rich stars has grown, we can re-examine the distribution of these objects. In Figure 12 we have gathered together data for a large number of C-rich stars from a number of surveys. Although the $\mathrm{T}_{e f f}$ and $\log g$ values for these stars have not been homogeneously determined, it seems clear that the a variety of $s$-process abundance levels for C-rich stars occur at all evolutionary states. This kind of plot does not by itself argue for different origins of the two groups of C-rich stars.

This review and others have documented the rapid evolution in our knowledge of the early chemical enrichment of our Galaxy. The stellar spectroscopic database is rich in data of low and high resolution. Atomic physicists have made significant improvements especially in theoretical computations for light elements and experimental data for the heavier elements. The combination of these developments has substantially increased our understanding of the origin of the elements and Galactic chemical evolution, and will continue to do so in the future.

\section{ACKNOWLEDGMENTS}

We thank the US National Science Foundation for financial support of this research, under grants AST-0607708 to CS and AST-0506324 to JEL. It is a pleasure to acknowledge the contributions of our collaborators in our papers cited in this review.

\section{REFERENCES}

1. S. Feltzing, and G. Gonzalez, Astron. Astrophys. 367, 253-265 (2001) 
2. W. Aoki, A. Frebel, et al., Astrophys. J. 639, 897-917 (2006).

3. J. W. Chamberlain, and L. H. Aller, Astrophys. J. 114, 52-72 (1951).

4. G. Wallerstein, Astrophys. J. 6, 407-443 (1962)

5. R. Gratton, C. Sneden, and E. Carretta, Ann. Rev. Astron. Astrophys. 42, 385-440 (2004).

6. B. E. Reddy, et al., Mon. Not. Roy. Astron. Soc. 367, 1329-1366 (2006).

7. G. Tautvaisiene, et al., Astron. J. 127, 373-379 (2004).

8. J. G. Cohen, and J. Meléndez, Astron. J. 129, 303-329 (2005).

9. J. W. Lee, et al., Astron. J. 129, 251-266 (2005).

10. D. Yong, et al., Astron. Astrophys. 438, 875-888 (2005)

11. R. G. Gratton, et al., Astron. Astrophys. 455, 271-281 (2006).

12. J. W. Lee, M. López-Morales, and B. W. Carney, Astrophys. J. 646, L119-L122 (2006).

13. E. Carretta, et al., Astron. Astrophys. 464, 967-981 (2007).

14. G. Wallerstein, et al., Astron. J. 133, 1373-1382 (2007).

15. E. Carretta, et al., Astron. J. 122, 1469-1485 (2001).

16. J. A. Brown, G. Wallerstein, and D. Zucker, Astron. J. 114, 180-188 (1997)

17. J. G. Cohen, Astron. J. 127, 1545-1554 (2004).

18. W. E. Harris, Astrophys. J. 112, 1487-1488 (1996).

19. B. Chaboyer, P. Demarque, and A. Sarajetini, Astrophys. J. 459, 558-569 (1996).

20. J. W. Lee, and B. W. Carney, Astron. J. 124, 1151-1527 (2002).

21. A. McWilliam, et al., Astron. J. 109, 2757-2799 (1995).

22. R. Cayrel, et al., Astron. Astrophys. 416, 1117-1138 (2004).

23. T. Sivarani, et al., Astron. Astrophys. 459, 125-135 (2006).

24. P. Bonifacio, et al., Astron. Astrophys. 462, 851-864 (2007).

25. J. Fulbright, Astron. J. 120, 1841-1852 (2007).

26. B. E. Reddy, et al., Mon. Not. Roy. Astron. Soc. $\mathbf{3 4 0 ,}$ 304-340 (2003).

27. B. W. Carney, et al., Astron. J. 114, 363-375 (1997)

28. I. I. Ivans, et al., Astrophys. J. 592, 906-934 (2003).

29. G. W. Preston, and C. Sneden, Astron. J. 120, 1014-1055 (2000).

30. J. G. Cohen, et al., Astrophys. J. 659, L161-L164 (2007).

31. W. Aoki, et al., Astrophys. J. 660, 747-761 (2007).

32. G. Wallerstein, et al., Astrophys. J. 137, 280-300 (1963).

33. E. Depagne, et al., Astron. Astrophys. 390, 187-198 (2002).

34. P. François, Astron. Astrophys. 160, 264-276 (1986).

35. S. G. Ryan, J. E. Norris, and T. C. Beers, Astron. J. 471 , 254-278 (1996).

36. K. Butler, C. Mendoza, and C. J. Zeippen, J. Phys. B. 26, 4409-4423 (1993)

37. C. Froese Fischer, G. Tachiev, and A. Irimia, At. Dat. Nuc. Dat. Tab. 92, 607-812 (2006).

38. M. Aldenius, et al., Astron. Astrophys. 461, 767-773 (2007).

39. C. Mendoza, et al., J. Phys. B 28, 3485-3504 (1995).

40. P. Hannaford, and R. M. Lowe, J. Phys. B 14, L5-L9 (1981).

41. S. Salih, and J. E. Lawler, Phys. Rev. A 28, 3653-3655 (1983).
42. D. Havey, C. Balling, and J. Wright, J. Opt. Soc. Am. 67, 491-493 (1977)

43. G. Smith, and D. S. J. Raggett, J. Phys. B 14, 4015-4024 (1981).

44. G. W. Preston, et al., Astron. J. 132, 85-110 (2006)

45. J. G. Cohen, et al., Astrophys. J. 612, 1107-1135 (2004).

46. M. Asplund, Astron. Astrophys. 435, 339-340 (2005).

47. A. E. Garcia Pérez, et al., Astron. Astrophys. 451, 621-642 (2006)

48. I. Ramirez, C. Allende Prieto, and D. L. Lambert, Astron. Astrophys. 465, 271-289 (2007).

49. L. Mashonkina, A. J. Korn, and N. Przybilla, Astron Astrophys. 461, 261-275 (2007)

50. C. Abia, and L. Mashonkina, Mon. Not. Roy. Astron. Soc. 350, 1127-1140 (2004).

51. A. G. W. Cameron, Astrophys. Space Sci. 82, 123-131 (1982).

52. F. Käppeler, et al., Rep. Prog. Phys. 52, 945-1013 (1989).

53. J. Simmerer, et al., Astrophys. J. 617, 1091-1114 (2004)

54. A. G. W. Cameron, Chalk river laboratory report crl-41, Tech. rep., Chalk River, Canada (1957)

55. I. I. Ivans, et al., Astrophys. J. 645, 613-633 (2006)

56. C. Sneden, et al., Astrophys. J. 591, 936-953 (2003).

57. J. Westin, et al., Astrophys. J. 530, 783-799 (2000).

58. J. J. Cowan, et al., Astrophys. J. 572, 861-879 (2002).

59. M. Spite, and F. Spite, Astron. Astrophys. 230, 23-31 (1978).

60. J. W. Truran, Astron. Astrophys. 97, 391-393 (1981).

61. R. Griffin, et al., Mon. Not. Roy. Astron. Soc. 198, 637-658 (1982)

62. C. Sneden, and M. Parthasarathy, Astrophys. J. 267, 757-778 (1983)

63. C. Sneden, and C. A. Pilachowski, Astrophys. J. 288, L55-L58 (1985).

64. K. K. Gilroy, et al., Astrophys. J. 327, 298-320 (1988)

65. C. Sneden, et al., Astrophys. J. 431, L27-L30 (1994).

66. C. Sneden, et al., Astrophys. J. 467, 819-840 (1996).

67. J. N. Norris, S. G. Ryan, and T. C. Beers, Astrophys. J. 488, 350-363 (1997).

68. C. Sneden, et al., Astrophys. J. 533, L139-L412 (2000).

69. S. Honda, et al., Astrophys. J. 607, 474498 (2004)

70. V. Hill, et al., Astron. Astrophys. 387, 560-579 (2002)

71. J. E. Lawler, et al., Astrophys. J. Supp. 162, 227-260 (2006).

72. E. A. Den Hartog, et al., Astrophys. J. Supp. 167, 292-314 (2006)

73. K. Lodders, Astrophys. J. 591, 1220-1247 (2003)

74. O. Hauge, Solar Phys. 11, 17-21 (1972).

75. J. E. Lawler, et al., Astrophys. J. 563, 1075-1088 (2001).

76. C. Sneden, et al., Astrophys. J. 556, L25-L28 (2002).

77. W. Aoki, et al., Astrophys. J. 586, 506-511 (2003).

78. P. Magain, Astron. Astrophys. 297, 686 694 (1995)

79. D. L. Lambert, and C. Allende Prieto, Mon. Not. Roy. Astron. Soc. 335, 325-334 (2002).

80. T. Masseron, et al., Astron. Astrophys. 455, 1059-1072 (2006).

81. M. Lundqvist, G. M. Wahlgren, and V. Hill, Astron Astrophys. 463, 693-702 (2007).

82. I. U. Roederer, et al., Astrophys. J. p. submitted (2007)

83. H. R. Butcher, Nature 328, 127-131 (1987).

84. P. François, M. Spite, and F. Spite, Astron. Astrophys. 274, 821-824 (1993). 
85. J. J. Cowan, et al., Astrophys. J. 521, 194-205 (1999).

86. J. J. Cowan, and C. Sneden, Nature 440, 1151-1156 (2006).

87. S. Goriely, and M. Arnould, Astron. Astrophys. 379, 1113-1122 (2001).

88. R. Cayrel, et al., Nature 409, 691-692 (2001).

89. A. Frebel, et al., Astrophys. J. 660, L117-L120 (2007).

90. P. S. Barklem, et al., Astron. Astrophys. 439, 129-151 (2005).

91. K.-L. Kratz, et al., Astrophys. J. 662, 39-52 (2007).

92. J. J. Cowan, et al., Astrophys. J. 627, 238-250 (2005).

93. E. Biémont, et al., Astron. Astrophys. 312, 116-122 (2000).

94. S. Ivarsson, et al., Astron. Astrophys. 409, 1141-1149 (2003).

95. E. A. Den Hartog, et al., Astrophys. J. 619, 639-655 (2005).

96. M. Lundqvist, et al., Astron. Astrophys. 450, 407-413 (2006).

97. J. E. Lawler, et al., Astrophys. J. Supp. 169, 120-136 (2007).

98. J. A. Johnson, and M. Bolte, Astrophys. J. 579, 616-625 (2002).

99. W. Aoki, et al., Astrophys. J. 632, 611-637 (2005).

100. G. J. Wasserburg, M. Busso, and R. Gallino, Astrophys. J. 466, L109-L113 (1996).

101. C. Travaglio, et al., Astrophys. J. 601, 864-884 (2004).

102. A. Gallagher, Phys. Rev. 157, 24-30 (1967).

103. P. Hannaford, et al., Solar Phys. 261, 736-746 (1982).

104. G. Ljung, Astron. Astrophys. 456, 1181-1185 (2006).

105. G. Malcheva, et al., Mon. Not. Roy. Astron. Soc. 367, $754-762(2006)$

106. I. I. Ivans, et al., Astrophys. J. 111, 111-111 (2005).

107. J. G. Cohen, et al., Astrophys. J. 633, L109-L112 (2005).

108. S. Lucatello, et al., Astrophys. J. 652, L37-40 (2006).

109. T. Kipper, and U. G. Jorgensen, Astron. Astrophys. 290, 148-158 (1994).

110. T. Kipper, et al., Astron. Astrophys. 306, 489-500 (1996).

111. S. Junqueira, and C. B. Pereira, Astron. J. 122, 360-369 (2001).

112. G. W. Preston, and C. Sneden, Astron. J. 122, 1545-1560 (2001).

113. W. Aoki, et al., Pub. Astron. Soc. Japan 54, 427-449 (2002).

114. W. Aoki, et al., Pub. Astron. Soc. Japan 54, 933-949 (2002).

115. J. G. Cohen, et al., Astrophys. J. 588, 1082-1098 (2003).

116. S. Lucatello, et al., Astron. J. 125, 875-893 (2003).

117. S. Van Eck, et al., Astron. Astrophys. 404, 291-299 (2003).

118. C. Sneden, G. W. Preston, and J. J. Cowan, Astrophys. J. 591, 936-953 (2003)

119. B. Barbuy, et al., Astron. Astrophys. 429, 1031-1042 (2005).

120. J. G. Cohen, et al., Astron. J. 132, 137-160 (2006).

121. A. Goswami, et al., Mon. Not. Roy. Astron. Soc. 372, 343-356 (2006).

122. K. Jonsell, et al., Astron. Astrophys. 451, 651-670 (2006).

123. W. Aoki, et al., Astrophys. J. 655, 492-521 (2007).

124. G. W. Preston, et al., Astron. J. 132, 1714-1724 (2006).

125. I. B. Thompson, et al., Astron. J. p. submitted (2007)
126. I. U. Roederer, et al., Astron. J. p. to be submitted (2007).

127. R. D. McClure, J. M. Fletcher, and J. M. Nemec, Astrophys. J. 238, L35-L38 (1980)

128. R. D. McClure, Astrophys. J. 280, L31-L34 (1984).

129. R. D. McClure, and A. W. Woodsworth, Astrophys. J. 352, 709-723 (1990).

130. S. Lucatello, et al., Astrophys. J. 625, 825-832 (2006).

131. S. G. Ryan, et al., Astrophys. J. 635, 349-354 (2005). 